\title{
Diffuse Large B-cell Lymphoma during Corticosteroid Therapy for TAFRO Syndrome
}

\author{
Eiko Ohya, Minoru Mizutani, Haruna Sakaguchi and Takao Sekine
}

\begin{abstract}
Thrombocytopenia, anasarca, myelofibrosis, renal dysfunction and organomegaly (TAFRO) syndrome is a variant of Castleman's disease recently identified in Japan. A 73-year-old man was diagnosed with TAFRO syndrome according to clinical findings, and his symptoms improved after corticosteroid therapy. Ten months later, lymphadenopathy worsened during tapering of corticosteroids. Histological findings of abdominal lymph nodes showed diffuse large B-cell lymphoma. After 6 cycles of R-CHOP therapy, he has remained in sustained complete remission. This is a rare case of the development of malignant lymphoma during the treatment of TAFRO syndrome, which suggests an association between diffuse large B-cell lymphoma and TAFRO syndrome.
\end{abstract}

Key words: TAFRO syndrome, corticosteroid therapy, lymphadenopathy, diffuse large B-cell lymphoma

(Intern Med 55: 2861-2867, 2016)

(DOI: 10.2169/internalmedicine.55.6455)

\section{Introduction}

Castleman's disease (CD) is a polyclonal lymphoproliferative disorder which was first reported by Dr. Benjamin Castleman in 1956 (1). CD is histopathologically classified into the hyaline vascular (HV) type, plasma cell (PC) type and mixed type (2). Frizzera and colleagues reported three variant types of CD: localized CD of HV type, localized CD of PC type, and multicentric Castleman's disease (MCD) $(2,3)$. MCD is clinicopathologically classified into the idiopathic plasmacytic lymphadenopathy (IPL) type and non-IPL type. Recently, some features specific to Japanese patients with MCD were revealed. In contrast to Western patients, most Japanese patients with idiopathic MCD are negative for both human herpesvirus-8 (HHV-8) and human immunodeficiency virus (HIV) $(4,5)$. Secondary MCD is related to HIV infection, IgG4-related disease, malignant lymphoma, plasma cell dyscrasia and systemic inflammatory disease (5-9). Furthermore, a variant of MCD with various systemic symptoms, thrombocytopenia, anasarca, myelofibrosis, renal dysfunction and organomegaly (TAFRO) syndrome, was first reported by Takai et al. (10). Kojima et al. reported Japanese MCD cases with effusion and thrombocytopenia
(Castleman-Kojima disease) (6). The histopathologic diagnosis of the affected lymph nodes is reportedly of the mixed type and less frequently of the HV type. Various corticosteroids, chemotherapy such as CHOEP (cyclophosphamide, doxorubicin hydrochloride, vincristine, etoposide, and prednisolone) and CVP (cyclophosphamide, vincristine, and prednisolone), as well as tocilizumab, cyclosporine $\mathrm{A}$ and rituximab have been used to treat TAFRO syndrome (11-16). Each case has had a different clinical course and outcomes and, to date, there is no consensus on the therapeutic strategies for TAFRO syndrome.

We herein report a rare case of a patient with TAFRO syndrome who developed diffuse large B-cell lymphoma (DLBCL) during successful corticosteroid therapy for constitutional symptoms of TAFRO syndrome.

\section{Case Report}

A 73-year-old man had noticed dyspnea on exertion and fatigue. Three weeks later he was admitted to our hospital because of anemia, thrombocytopenia and renal dysfunction. $\mathrm{He}$ had a past medical history of diabetes mellitus and uveitis. Upon admission, he reported having gained $5 \mathrm{~kg}$ in body weight within 1 month. He had a continuous fever 
Table. Laboratory Data on Admission.

\begin{tabular}{|c|c|c|c|c|c|c|}
\hline \multicolumn{2}{|c|}{ Complete blood cell count } & \multicolumn{2}{|l|}{ Biochemistry } & \multicolumn{2}{|l|}{ Immunologic test } & \multirow{2}{*}{$\frac{(\text { reference range })}{(870-1,700)}$} \\
\hline White blood cell & $6,700 / \mu \mathrm{L}$ & Total protein & $6.6 \mathrm{~g} / \mathrm{dL}$ & $\operatorname{IgG}$ & $1,678 \mathrm{mg} / \mathrm{dL}$ & \\
\hline Red blood cell & $359 \times 10^{4} / \mu \mathrm{L}$ & Albumin & $2.4 \mathrm{~g} / \mathrm{dL}$ & IgA & $309 \mathrm{mg} / \mathrm{dL}$ & $(110-410)$ \\
\hline Hemoglobin & $9.8 \mathrm{~g} / \mathrm{dL}$ & Total bilirubin & $1.1 \mathrm{mg} / \mathrm{dL}$ & IgM & $468 \mathrm{mg} / \mathrm{dL}$ & $(35-220)$ \\
\hline Hematocrit & $29.9 \%$ & AST & $16 \mathrm{IU} / \mathrm{L}$ & $\mathrm{CH} 50$ & $60 \mathrm{U} / \mathrm{mL}$ & $(30-45)$ \\
\hline Platelet & $1.7 \times 10^{4} / \mu \mathrm{L}$ & ALT & $9 \mathrm{IU} / \mathrm{L}$ & ANA & $\times 40$ speckled & $(<\times 40)$ \\
\hline \multicolumn{2}{|l|}{ Coagulation test } & LDH & $287 \mathrm{IU} / \mathrm{L}$ & anti-ds-DNA IgG & $<10 \mathrm{IU} / \mathrm{mL}$ & $(<10)$ \\
\hline PT & $14.7 \mathrm{~s}$ & ALP & $371 \mathrm{IU} / \mathrm{L}$ & PR3-ANCA & $<1.0 \mathrm{U} / \mathrm{mL}$ & $(<1.0)$ \\
\hline PT-INR & 1.23 & Glucose & $118 \mathrm{mg} / \mathrm{dL}$ & MPO-ANCA & $<1.0 \mathrm{U} / \mathrm{mL}$ & $(<1.0)$ \\
\hline APTT & $38.5 \mathrm{~s}$ & BUN & $27 \mathrm{mg} / \mathrm{dL}$ & aCL- $\beta 2 \mathrm{GP} 1 \mathrm{I}$ & $<1.3 \mathrm{U} / \mathrm{mL}$ & $(<3.5)$ \\
\hline Fibrinogen & $478 \mathrm{mg} / \mathrm{dL}$ & Creatinine & $1.77 \mathrm{mg} / \mathrm{dL}$ & Coombs test & - & $(-)$ \\
\hline FDP & $18.4 \mu \mathrm{g} / \mathrm{mL}$ & CRP & $9.23 \mathrm{mg} / \mathrm{dL}$ & PA-IgG & $208 \mathrm{ng} / 10^{7}$ cells & $(\leq 46)$ \\
\hline \multicolumn{2}{|l|}{ Urine test } & Free T4 & $1.23 \mathrm{ng} / \mathrm{dL}$ & Cytokines & & \\
\hline Protein & $2+$ & TSH & $878 \mu \mathrm{IU} / \mathrm{mL}$ & sIL-2R & $1,769 \mathrm{U} / \mathrm{mL}$ & $(122-496)$ \\
\hline Sugar & - & Ferritin & $551.1 \mathrm{ng} / \mathrm{mL}$ & IL-6 & $5.3 \mathrm{pg} / \mathrm{mL}$ & $(\leq 4.0)$ \\
\hline Occult blood & + & \multicolumn{2}{|l|}{ Virologic test } & & & \\
\hline \multirow[t]{2}{*}{ Granular cast } & $2+$ & \multicolumn{2}{|l|}{ HIV-Antibody } & & & \\
\hline & & \multicolumn{2}{|l|}{ HHV-8-DNA } & & & \\
\hline
\end{tabular}

PT: prothrombin time, PT-INR: prothrombin time-international normalized ratio, APTT: activated partial thromboplastin time, FDP: fibrinogen degradation products, AST: aspartate aminotransferase, ALT: alanine aminotransferase, LDH: lactate dehydrogenase, ALP: alkaline phosphatase, BUN: blood urea nitrogen, CRP: C-reactive protein, TSH: thyroid-stimulating hormone, HIV: human immunodeficiency virus, HHV-8: human herpesvirus-8, ANA: antinuclear antibody, anti-dsDNA IgG: anti-double-stranded DNA IgG antibody, PR3-ANCA: proteinase 3-anti neutrophil cytoplasmic antibody, MPOANCA: myeloperoxidase-anti neutrophil cytoplasmic antibody, aCL- $\beta 2 \mathrm{GPI}$ : anti-cardiolipin- $\beta 2$ glycoprotein I complex antibodies, PA-IgG: platelet-associated IgG, SIL-2R: soluble interleukin-2 receptor, IL-6: interleukin-6

ranging between $37.5^{\circ} \mathrm{C}$ and $38^{\circ} \mathrm{C}$, abdominal distention and pitting edema in the lower extremities. Peripheral lymph nodes were not palpable. As shown in Table, his laboratory data showed microcytic anemia, severe thrombocytopenia, and elevated C-reactive protein (CRP), lactate dehydrogenase (LDH), alkaline phosphatase (ALP), soluble interleukin-2 receptor and ferritin levels. Hypoalbuminemia, renal dysfunction, polyclonal hyperglobulinemia, proteinuria and microscopic hematuria were revealed. The serum level of platelet-associated $\operatorname{IgG}(\mathrm{PA}-\mathrm{IgG})$ was elevated, although other autoantibodies were negative. Serum antibodies against hepatitis $\mathrm{B}$ and $\mathrm{C}$ virus, HIV, human T-lymphotropic virus-1 and HHV-8 were all negative. The serum level of IL-6 was slightly elevated. Computed tomography (CT) revealed mediastinal and para-aortic lymphadenopathy $(<1.5 \mathrm{~cm}$ in diameter), bilateral pleural effusions, ascites and splenomegaly (Fig. 1A-D). The ascites contained exudate alone without tumor cells. An ${ }^{18} \mathrm{~F}$-fluorodeoxyglucose positron emission tomography (FDG-PET)-CT scan was not performed because his general condition rapidly deteriorated. We could not perform a lymph node biopsy because he had no peripheral lymphadenopathy and his thrombocytopenia was severe. Bone marrow aspiration resulted in a dry tap. A bone marrow biopsy exhibited a mixture of hypocellular and hyper- cellular marrow with increased megakaryocytes and mild fibrosis without pathologic cells, dysplastic changes or hemophagocytosis (Fig. 2). Oliguria developed and hemodialysis was initiated. Because his clinical features were thought to be compatible with TAFRO syndrome, corticosteroid therapy $(0.5 \mathrm{mg} / \mathrm{kg} / \mathrm{day}$ of prednisolone) was started. The fever, edema, pleural effusion, ascites, anemia and renal dysfunction immediately improved, and hemodialysis was discontinued after a week. However, thrombocytopenia persisted and the patient was refractory to platelet transfusion. The platelet count increased after eltrombopag was administered (12.5 mg daily) for 2 weeks and then tapered. He was subsequently discharged and prednisolone was carefully tapered by $2.5 \mathrm{mg} / \mathrm{body} /$ day per month to $5 \mathrm{mg} /$ body/day.

Although the patient's initial symptoms such as edema, pleural effusion, ascites, anemia, thrombocytopenia and renal dysfunction disappeared 10 months after the disease onset, the CRP level slightly increased. A CT scan demonstrated abdominal and pelvic lymphadenopathy and a left adrenal mass (Fig. 1E-H). Increased FDG uptakes in the right cervical, mediastinal, abdominal para-aortic, iliac lymph nodes, left shoulder [maximum standardized uptake value (SUVmax) 39.2], left adrenal mass (SUVmax 33.0) and liver (SUVmax 9.3) were observed by PET-CT (Fig. 3). A 

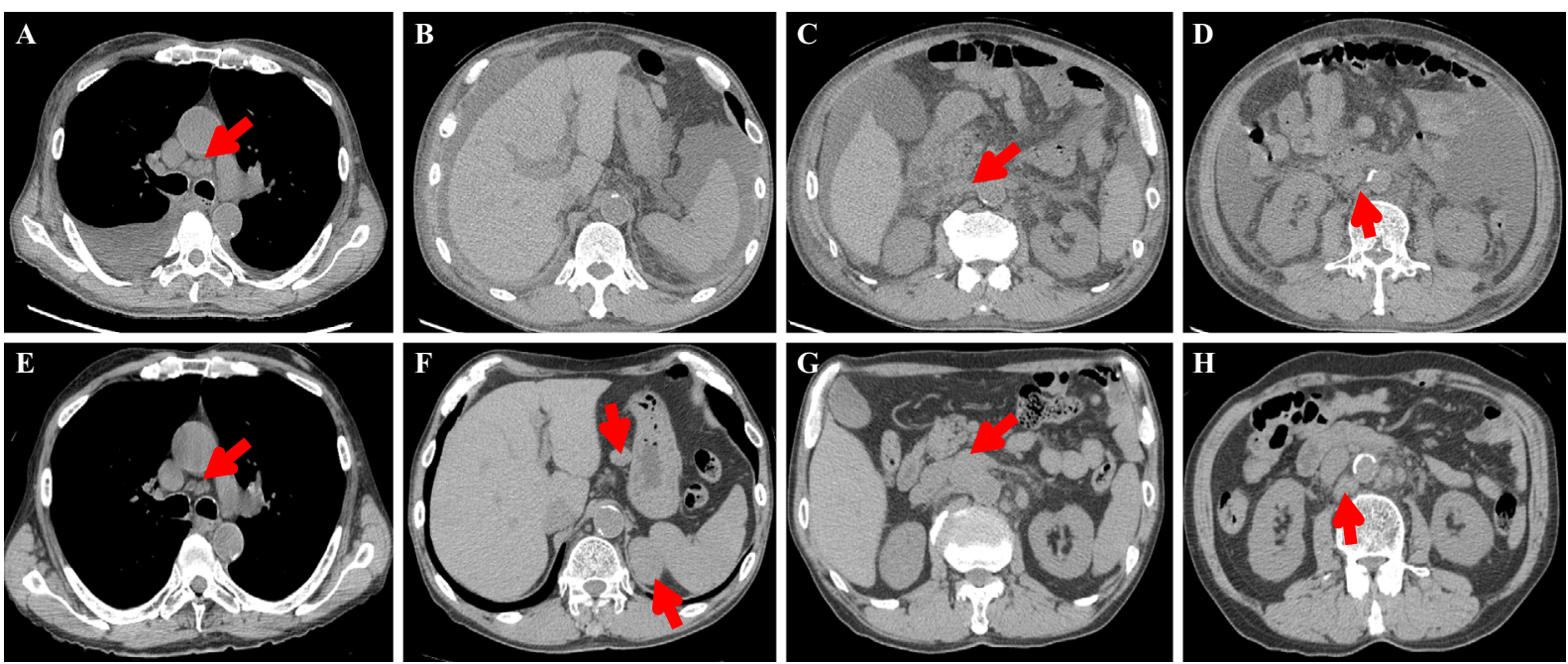

Figure 1. CT images on admission and 10 months after the disease onset. (A-D) Images on admission showed mediastinal and para-aortic lymphadenopathy $(<1.5 \mathrm{~cm}$ in diameter), bilateral pleural effusions, ascites and mild splenomegaly. (E-H) Ten months after the disease onset, abdominal and pelvic lymphadenopathy and a left adrenal mass were observed. There was no change in the size of the mediastinal lymph nodes. pleural effusion and ascites were not observed.
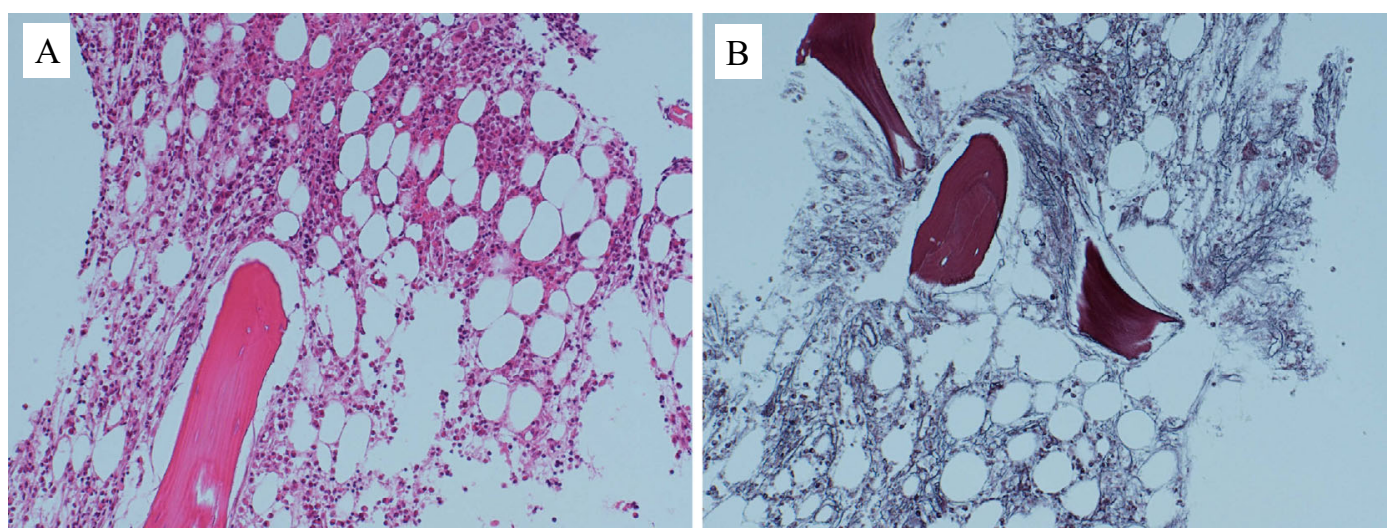

Figure 2. Histological findings of the bone marrow. (A) The mixture of hypocellular and hypercellular marrow with increased megakaryocytes was observed without pathologic cells, dysplastic changes or hemophagocytosis. Hematoxylin and Eosin staining, 100×. (B) Mild hyperplasia of reticulin fibrosis was observed. Silver stain, 100x.

biopsy of the right cervical lymph node showed follicular hyperplasia without neoplastic features (Fig. 4). Bone marrow aspiration again resulted in a dry tap and the histological findings of the bone marrow biopsy were the same as those in the initial examination. A cervical lymph node biopsy revealed follicular hyperplasia, which is not a characteristic histopathologic finding in TAFRO syndrome, however we suspected the recurrence of TAFRO syndrome clinically. Prednisolone was increased to $20 \mathrm{mg}$, but abdominal and pelvic lymphadenopathy deteriorated. Systemic edema, renal dysfunction due to bilateral hydronephrosis and left deep venous thrombosis developed. Neither high-dose steroid therapy (250 mg of intravenous methylprednisolone for 3 days followed by $40 \mathrm{mg}$ of dexamethasone for 4 days) nor $8 \mathrm{mg} / \mathrm{kg}$ of tocilizumab was effective. A surgical biopsy of the abdominal para-aortic lymph node and a core needle bi- opsy of the left shoulder mass were performed, and the histopathologic findings of both were consistent with nonHodgkin's lymphoma of diffuse large B-cell type (Fig. 5). According to these findings, the patient was diagnosed with DLBCL clinical stage IV, and a decreased dose of R-CHOP (rituximab $375 \mathrm{mg} / \mathrm{m}^{2}$, doxorubicin $50 \mathrm{mg} / \mathrm{m}^{2}$, cyclophosphamide $600 \mathrm{mg} / \mathrm{m}^{2}$, vincristine $1 \mathrm{mg} / \mathrm{body}$ and prednisolone $40 \mathrm{mg} / \mathrm{m}^{2}$ ) was administered in the first cycle. He developed septic shock caused by extended-spectrum betalactamase-producing Escherichia coli during neutropenia. The dose of CHOP was further reduced to $75 \%$ of the initial dose for the following 5 cycles. A CT scan after 2 cycles of chemotherapy revealed a marked regression of abdominal and pelvic lymphadenopathy and improved hydronephrosis. After 6 cycles of modified R-CHOP, PET-CT showed complete resolution of all FDG-avid lesions at the diagnosis and 

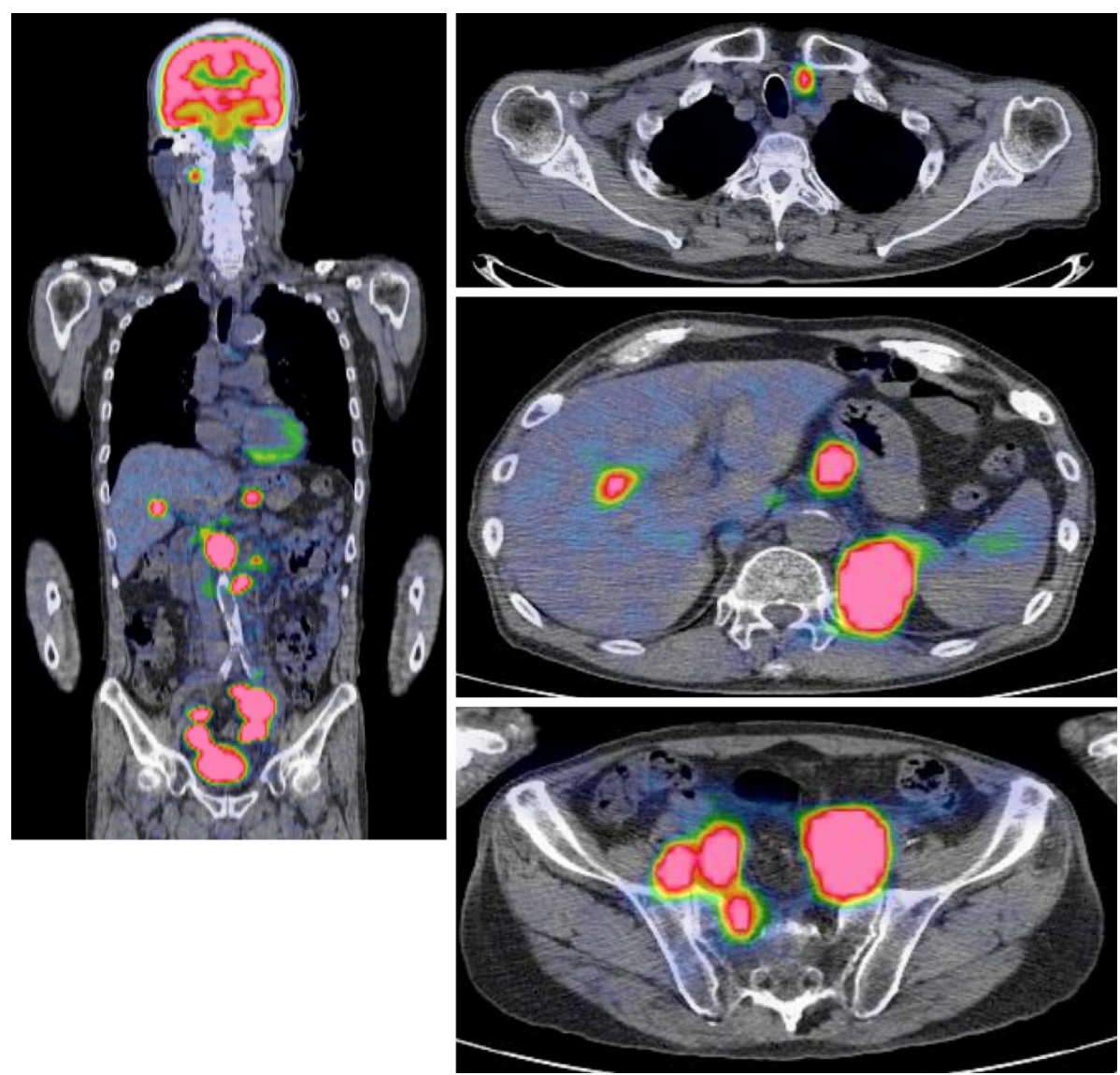

Figure 3. Positron emission tomography (PET) -CT imaging 10 months after the disease onset. ${ }^{18} \mathrm{~F}$ fluorodeoxyglucose uptake were observed in the right cervical, mediastinal, abdominal para-aortic, iliac lymph nodes, left shoulder [maximum standardized uptake value (SUVmax) 39.2], left adrenal mass (SUVmax 33.0) and liver (SUVmax 9.3).
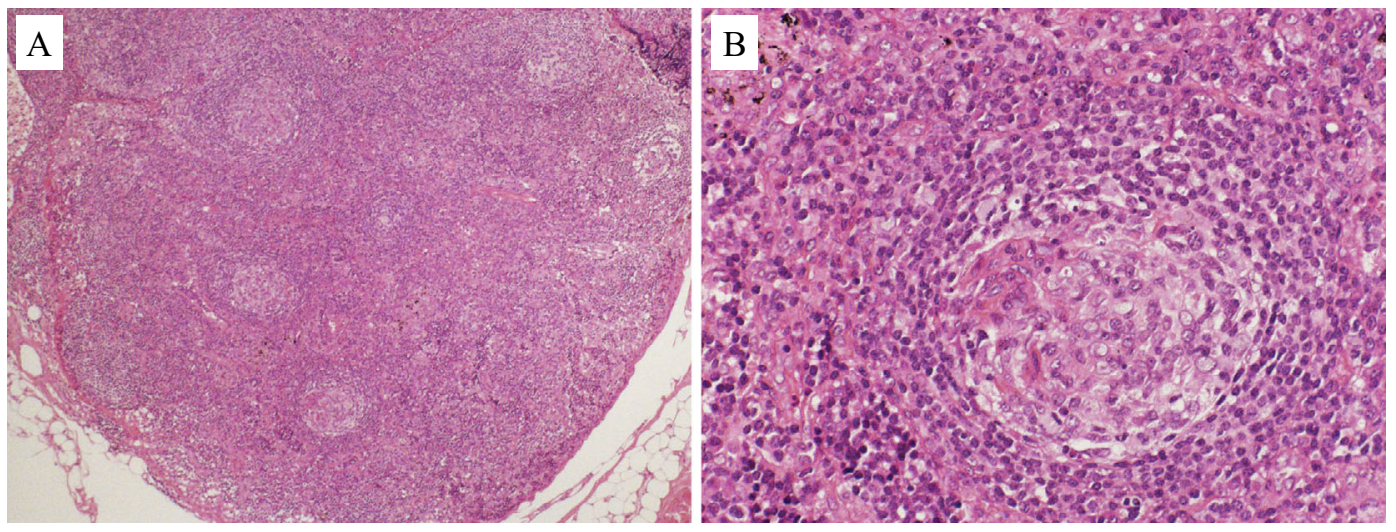

Figure 4. Histological findings of the right cervical lymph node. (A) Hematoxylin and Eosin (H\&E) staining, 40x. (B) H\&E staining, 200x. The lymph node showed hyperplasia of lymphoid follicles and non-atrophic germinal centers. Infiltration of plasma cells in the interfollicular area and proliferation of endothelial cells were unremarkable. Neoplastic features were not detected.

documented complete remission. The systemic symptoms at the onset of TAFRO syndrome, such as edema, pleural effusion, ascites and lymphadenopathy disappeared. Additionally, abnormalities such as anemia, thrombocytopenia, renal dysfunction and elevated CRP level were improved.

The patient currently remains well in complete remission without corticosteroid therapy.

\section{Discussion}

TAFRO syndrome (Castleman-Kojima disease) is a systemic inflammatory disorder characterized by a collection of 

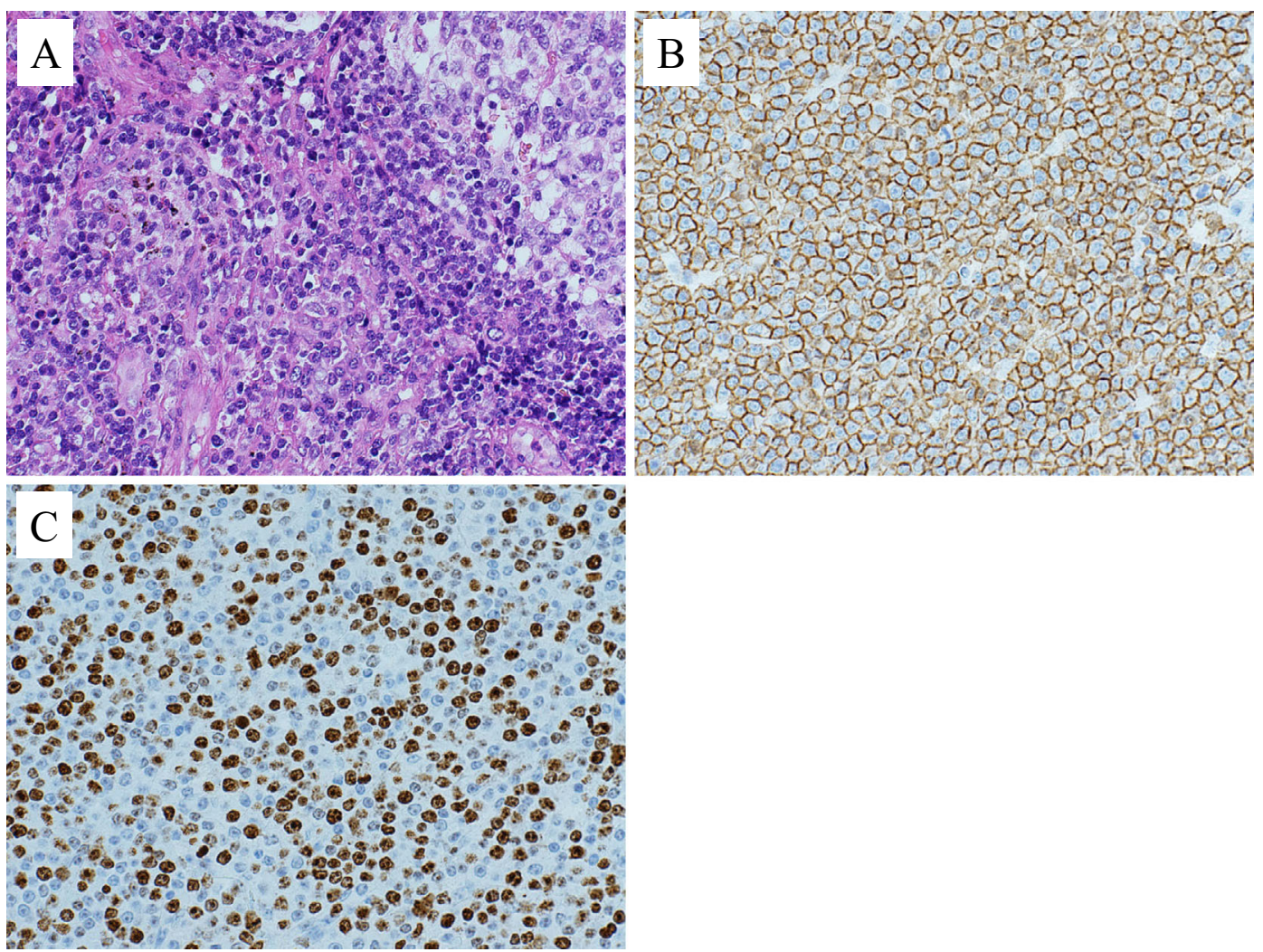

Figure 5. Histological and immunohistochemical findings of the abdominal lymph node. (A) Hematoxylin and Eosin staining, 200×. (B) CD20 immunostaining, 200×. (C) Ki-67 immunostaining, 200×. The basic structure of the lymph node had disappeared and diffuse proliferation of large atypical lymphoid cells with prominent nucleoli and frequent mitotic figures was demonstrated. These cells were positive for CD20, CD79a, B-cell lymphoma (BCL) -2, BCL-6, and multiple myeloma oncogene-1 and negative for CD3, CD5, CD10 and Epstein-Barr virus-encoded RNAs. The Ki-67 labeling index was elevated. These findings were consistent with DLBCL.

systemic symptoms, thrombocytopenia, ascites (anasarca), myelofibrosis, renal dysfunction and organomegaly. It was modified in a consensus conference on September 22, 2012 (9). Other characteristic clinical findings are microcytic anemia, elevated level of ALP, decreased level of LDH, positivity of autoantibodies (antinuclear antibody, rheumatoid factor, PA-IgG, anti-thyroid antibody and direct Coombs test) and elevated levels of IL- 6 and the vascular endothelial growth factor (VEGF) in the serum or effusions. This disease occurs in the middle-aged and elderly and is four times more common in women than in men. The lymphadenopathy is mild and generally measures less than 1.5 $\mathrm{cm}$ in diameter. For an accurate diagnosis, exclusion of lymphoma and other lymphoproliferative disorders by a lymph node biopsy is necessary. The histopathologic diagnoses include mixed type and, less frequently, HV type CD. The clinical course is indolent in many cases, but some patients' conditions rapidly worsen, in which case the prognosis is poor (9).

In the present case, we initially suspected that the systemic symptoms were caused by systemic lupus erythematosus (SLE) or angioimmunoblastic T-cell lymphoma (AITL). We first attempted to rule out SLE because while some clinical symptoms (pleural effusion, ascites, proteinuria, he- maturia and thrombocytopenia) met the classification criteria for SLE (17), anemia was not caused by hemolysis and no specific antibodies were detected. AITL also causes various systemic symptoms, such as a fever, effusion and immunological abnormalities, however, myelofibrosis is rarely observed $(18,19)$. Although AITL was not initially completely ruled out, lymphoma cells were not detected in the ascites or bone marrow. We also suspected MCD as the cause of the fever, lymphadenopathy and effusion. Finally, the patient was diagnosed with TAFRO syndrome. The serum level of LDH was slightly elevated, but other clinical features met the clinical criteria of TAFRO syndrome. The clinical course showed relatively rapid exacerbation and prompt treatment was required. Corticosteroid therapy was effective and, with the exception of ongoing thrombocytopenia, his condition improved. We speculated that thrombocytopenia was caused by an autoimmune mechanism and administered eltrombopag. After 2 weeks, the platelet count had increased and eltrombopag was discontinued. Thrombocytopenia gradually improved.

Ten months after the disease onset, lymphadenopathy deteriorated despite continuous corticosteroid therapy. A high FDG uptake of the right cervical lymph node noted, in contradiction with the histological finding of follicular hyperpla- 
sia. It was considered possible that the right cervical lymph node had DLBCL lesions, which was confirmed by a biopsy. We propose two possible explanations for the association of TAFRO syndrome with DLBCL. First, DLBCL may have initially existed and TAFRO syndrome occurred secondary to DLBCL. Although corticosteroid therapy successfully treated TAFRO syndrome, it might have been ineffective for DLBCL. Second, DLBCL may have developed from a lymphoid lesion of TAFRO syndrome due to the elevation of inflammatory cytokines. Hyper-IL-6 syndrome and other cytokine storms are considered to be involved in the onset of TAFRO syndrome (11). Inflammatory cytokines such as IL-6, VEGF, IL-10, and tumor necrosis factor are also reported to be associated with DLBCL (20-22). In the present case, it is possible that the elevation of inflammatory cytokines caused both TAFRO syndrome and DLBCL.

To the best of our knowledge, this is the first report of the development of malignant lymphoma from TAFRO syndrome. Some cases of malignant lymphoma that developed from MCD have been reported. Ogawa et al. reported large B-cell lymphoma arising in HHV-8-associated MCD in one patient with HIV infection (23). Large B-cell lymphoma in HHV-8 MCD is associated with monoclonal proliferation of HHV-8-infected lymphoid cells. Lymphoma development from MCD in HIV-negative patients has also been reported, however the status of HHV-8 activity and pathogenesis remain unclear $(24,25)$. Moreover, inflammatory cytokines may be related to the onset of malignant lymphoma in HHV-8-negative MCD.

It may be difficult to perform a lymph node biopsy in patients with TAFRO syndrome because of its aggressive clinical course. Severe thrombocytopenia and lymphadenopathy in the deep lesion make it hard to access. Thus, some cases require a prompt diagnosis and treatment without a biopsy. However, a different disease such as malignant lymphoma may develop during the treatment, which occurred in the present case. A histological diagnosis is indispensable for a definitive diagnosis and appropriate treatment. Therefore, it is necessary to attempt a lymph node biopsy even if treatment has started.

The pathophysiological etiology and therapeutic strategy for TAFRO syndrome are still unclear. A greater accumulation of cases is needed to determine the appropriate diagnosis and therapy.

\section{The authors state that they have no Conflict of Interest (COI).}

\section{Acknowledgement}

The authors thank Professor Yasufumi Masaki (Hematology and Immunology, Kanazawa Medical University) and Dr. Noriko Iwaki (Department of Internal Medicine, Keiju Kanazawa Hospital) for assistance with the pathologic diagnosis and fruitful discussions.

\section{References}

1. Castleman B, Iverson L, Menendez VP. Localized mediastinal lymph node hyperplasia resembling thymoma. Cancer 9: 822-830, 1956.

2. Frizzera G, Peterson BA, Bayrd ED, Goldman A. A systemic lymphoproliferative disorder with morphologic features of Castleman's disease: clinical findings and clinicopathologic correlations in 15 patients. J Clin Oncol 3: 1202-1206, 1985.

3. Frizzera G. Castleman's disease and related disorders. Semin Diadn Pathol 5: 346-364, 1988.

4. Suda T, Katano H, Delsol G, et al. HHV-8 infection status of AIDS-unrelated and AIDS-associated multicentric Castleman's disease. Pathol Int 51: 671-679, 2001.

5. Kojima M, Nakamura N, Tsukamoto N, et al. Clinical implications of idiopathic multicentric Castleman's disease among Japanese. A report of 28 cases. Int J Surg Pathol 16: 391-398, 2008.

6. Kojima M, Nakamura N, Tsukamoto N, et al. Multicentric Castleman's disease representing effusion at initial clinical presentation: clinicopathological study of seven cases. Lupus 20: 44-50, 2011.

7. Iwaki J, Kojima M, Tomita K, et al. A case of IgG4-related lung disease associated with multicentric Castleman's disease and lung cancer. Intern Med 49: 1287-1291, 2010.

8. Owens CL, Weir EG, Ali SZ. Cytopathologic findings in "POEMS" syndrome associated with Castleman disease. Diagn Cytopathol 35: 512-515, 2007.

9. Kawabata H, Takai K, Kojima M, et al. Castleman-Kojima disease (TAFRO syndrome): a novel systemic inflammatory disease characterized by a constellation of symptoms, namely, thrombocytopenia, ascites (anasarca), microcytic anemia, myelofibrosis, renal dysfunction, and organomegaly: a status report and summary of Fukushima (6 June, 2012) and Nagoya meetings (22 September, 2012). J Clin Exp Hematop 53: 57-61, 2013.

10. Takai K, Nikkuni K, Shibuya H, Hashidate H. Thrombocytopenia with mild bone marrow fibrosis accompanied by fever, pleural effusion, ascites and hepatosplenomegaly. Rinsho Ketsueki (Jap. J. Clin. Hemat.) 51: 320-325, 2010 (in Japanese, Abstract in English).

11. Masaki Y, Nakajima A, Iwao H, et al. Japanese variant of multicentric Castleman's disease associated with serositis and thrombocytopenia - a report with two cases: is TAFRO syndrome (Castleman-Kojima disease) a distinct clinicopathological entity? J Clin Exp Hematop 53: 79-85, 2013.

12. Iwaki N, Sato Y, Takata K, et al. Atypical hyaline vascular-type Castleman's disease with thrombocytopenia, anasarca, fever, and systemic lymphadenopathy. J Clin Exp Hematop 53: 87-93, 2013.

13. Inoue $\mathrm{M}$, Ankou $\mathrm{M}, \mathrm{Hua} J$, et al. Complete resolution of TAFRO syndrome (thrombocytopenia, anasarca, fever, reticulin fibrosis and organomegaly) after immunosuppressive therapies using corticosteroids and cyclosporine A: a case report. J Clin Exp Hematop 53: 95-99, 2013.

14. Tedesco S, Postacchini L, Manfredi L, et al. Successful treatment of a Caucasian case of multifocal Castleman's disease with TAFRO syndrome with a pathophysiology targeted therapy: a case report. Exp Hematol Oncol 4: 3, 2015.

15. Kawabata H, Kotani S, Matsumura Y, et al. Successful treatment of a patient with multicentric Castleman's disease who presented with thrombocytopenia, ascites, renal failure and myelofibrosis using tocilizumab, an anti-interleukin-6 receptor antibody. Intern Med 52: 1503-1507, 2013.

16. Ozawa $T$, Kosugi S, Kito M, et al. Efficacy of rituximab for a variant type of multicentric Castleman's disease termed the TAFRO syndrome. Rinsho Ketsueki (Jap. J. Clin. Hemat.) 55: 350-355, 2014 (in Japanese, Abstract in English).

17. Petri M, Orbai AM, Alarcón GS, et al. Derivation and validation of the systemic lupus international collaborating clinics classification criteria for systemic lupus erythematosus. Arthritis Rheum 64: 2677-2686, 2012.

18. Matsui K, Adachi M, Tominaga T, Shinohara K, Kamei T. Angio- 
immunoblastic $\mathrm{T}$ cell lymphoma associated with reversible myelofibrosis. Intern Med 47: 1921-1924, 2008.

19. Sekiguchi Y, Matsuzawa N, Shimada A, et al. Angioimmunoblastic T-cell lymphoma with intramedullary production of plateletderived growth factor and possibly complicating myelofibrosis: report of a case with review of the literature. Int J Hematol 98: 250257, 2013.

20. Charbonneau B, Maurer MJ, Ansell SM, et al. Pretreatment circulating serum cytokines associated with follicular and diffuse large B-cell lymphoma: a clinic-based case-control study. Cytokine 60: 882-889, 2012.

21. Conroy SM, Maskarinec G, Morimoto Y, et al. Non-Hodgkin lymphoma and circulating markers of inflammation and adiposity in a nested case-control study: the multiethnic cohort. Cancer Epidemiol Biomarkers Prev 22: 337-347, 2013.

22. Hosgood HD 3rd, Au WY, Kim HN, et al. IL10 and TNF variants and risk of non-Hodgkin lymphoma among three Asian populations. Int J Hematol 97: 793-799, 2013.

23. Ogawa Y, Watanabe D, Hirota K, et al. Rapid multiorgan failure due to large B-cell lymphoma arising in human herpesvirus-8associated multicentric Castleman's disease in a patient with human immunodeficiency virus infection. Intern Med 53: 2805-2809, 2014.

24. Advani R, Warnke R, Rosenberg S. Treatment of multicentric Castleman's disease complicated by the development of nonHodgkin's lymphoma with high-dose chemotherapy and autologous peripheral stem-cell support. Ann Oncol 10: 1207-1209, 1999.

25. Park J, Lee JE, Kim M, et al. Discordant lymphocyte-depleted classical Hodgkin's and peripheral T-cell lymphoma arising in a patient 11 years after diagnosis of multicentric Castleman's disease. Int J Hematol 98: 114-121, 2013.

The Internal Medicine is an Open Access article distributed under the Creative Commons Attribution-NonCommercial-NoDerivatives 4.0 International License. To view the details of this license, please visit (https://creativecommons.org/licenses/ by-nc-nd/4.0/).

(C) 2016 The Japanese Society of Internal Medicine http://www.naika.or.jp/imonline/index.html 